\title{
Achieving high effective $Q$-factors in ultra-high vacuum dynamic force microscopy
}

\author{
Jannis Lübbe ${ }^{1}$, Lutz Tröger ${ }^{1}$, Stefan Torbrügge ${ }^{1,4}$, Ralf Bechstein ${ }^{1,5}$, \\ Christoph Richter ${ }^{2}$, Angelika Kühnle ${ }^{3}$ and Michael Reichling ${ }^{1}$ \\ ${ }^{1}$ Fachbereich Physik, Universität Osnabrück, Barbarastraße 7, 49076 Osnabrück, Germany \\ ${ }^{2}$ Nanoworld Services GmbH, Schottkystraße 10, 91058 Erlangen, Germany \\ ${ }^{3}$ Institut für Physikalische Chemie, Johannes Gutenberg-Universität Mainz, Jakob-Welder Weg 11, \\ 55099 Mainz, Germany \\ E-mail: reichling@uos.de
}

Received 5 August 2010, in final form 22 September 2010

Published 4 November 2010

Online at stacks.iop.org/MST/21/125501

\begin{abstract}
The effective $Q$-factor of the cantilever is one of the most important figures-of-merit for a non-contact atomic force microscope (NC-AFM) operated in ultra-high vacuum (UHV). We provide a comprehensive discussion of all effects influencing the $Q$-factor and compare measured $Q$-factors to results from simulations based on the dimensions of the cantilevers. We introduce a methodology to investigate in detail how the effective $Q$-factor depends on the fixation technique of the cantilever. Fixation loss is identified as a most important contribution in addition to the hitherto discussed effects and we describe a strategy for avoiding fixation loss and obtaining high effective $Q$-factors in the force microscope. We demonstrate for room temperature operation, that an optimum fixation yields an effective $Q$-factor for the NC-AFM measurement in UHV that is equal to the intrinsic value of the cantilever.
\end{abstract}

Keywords: cantilever, $Q$-factor, mounting loss, force microscopy, NC-AFM

(Some figures in this article are in colour only in the electronic version)

\section{Introduction}

Scanning force microscopy operated in the non-contact mode (NC-AFM) has become a standard tool for atomic scale surface characterization [1, 2] and is specifically well suited for imaging and manipulation on electrically insulating substrates [3-10]. Highest resolution measurements are obtained in ultra-high vacuum (UHV) using the frequency modulation method (FM-AFM) [11]. In this mode, the tip is periodically approached to the surface by oscillating the cantilever at its resonance frequency $f_{0}$. Upon close approach of the tip to the surface, the resonance frequency is shifted due to the

\footnotetext{
4 Present address: SPECS GmbH, Voltastrasse 5, 13355 Berlin, Germany.

5 Present address: Department of Physics and Astronomy, Interdisciplinary Nanoscience Center (iNANO), University of Aarhus, Ny Munkegade, 8000 Aarhus C, Denmark.
}

interaction of the tip with the surface. The resonance frequency shift $\Delta f$ of the cantilever oscillation is a measure of the forces acting between the tip and the surface. Considering thermal excitation as the source of fluctuations limiting the detection, the minimum detectable force gradient $\delta F_{\min }^{\prime}$ is defined via the minimum detectable frequency shift $\delta f$ as

$$
\delta F_{\text {min }}^{\prime}=\frac{2 k \delta f}{f_{0}}=\sqrt{\frac{4 k k_{\mathrm{B}} T B}{\pi Q f_{0} A^{2}}},
$$

where $k_{\mathrm{B}}, T, A$ and $B$ denote the Boltzmann constant, temperature, oscillation amplitude and detection bandwidth, respectively, $k$ is the spring constant of the cantilever and $Q$ its quality factor commonly referred to as the $Q$-factor [11]. From equation (1) it is evident that using high- $Q$ cantilevers improves the force sensitivity in a non-contact atomic force microscope. For atomic resolution measurements in UHV, 
cantilevers with $Q$-factors exceeding 10000 are typically used [2]. Therefore, it is desirable to measure the $Q$-factor of the cantilever as produced and to explore how the effective $Q$-factor relevant for NC-AFM measurements may change through steps of handling and mounting in the UHV force microscope. Here, we entirely focus on the $Q$-factor of the oscillation of the first eigenmode of the cantilever, although investigating the $Q$-factor of higher eigenmodes is of interest for advanced force microscopy techniques [12-17].

To facilitate testing of cantilevers prior to their use in the force microscope, we designed a test setup allowing the measurement of $Q$-factors in a separate vacuum chamber without irreversibly gluing cantilevers to a specific cantilever holder as used in the NC-AFM. We compare $Q$-factors measured in the test setup to $Q$-factors of the same cantilevers measured in a force microscope, and investigate in detail differences in the measured values depending on the fixation technique of the cantilever support chip on the cantilever holder. Furthermore, we investigate the influence of the fixation of the cantilever holder in the AFM systems on the effective $Q$-factor. Comparative measurements are performed in two systems, namely a modified Omicron UHV AFM/STM, further on referred to as system A [18, 19], and an Omicron VTAFM 25, further on referred to as system $B^{6}$. We use uncoated silicon cantilevers with resonance frequencies of about $75 \mathrm{kHz}$ $(\text { type } \mathrm{FM})^{7}$ and about $300 \mathrm{kHz}$ (type $\left.\mathrm{NCH}\right)^{7}$. Experiments are complemented by a comparison of measured $Q$-factors to predictions by analytical formulae relating the $Q$-factor to geometrical parameters of the cantilever. As illustrated in figure 1(a), cantilevers are rods of length $l$ and thickness $t$ and generally have a trapezoidal cross-section. For calculations they are, however, assumed as a rectangular bar with a width $w$ that is equal to the mean width of the trapezoidal rod.

The $Q$-factor of a damped system is defined as the ratio of the energy $W$ stored in the oscillating system to the energy $\Delta W$ dissipated per cycle [20]:

$$
Q=\frac{2 \pi W}{\Delta W} .
$$

A high quality factor results in a narrow resonance peak that is generally described by the following expression for the frequency-dependent amplitude $A$ :

$$
|A|=\frac{\left|A_{\mathrm{exc}}\right|}{\sqrt{\left(1-f_{\mathrm{exc}}^{2} / f_{0}^{2}\right)^{2}+f_{\mathrm{exc}}^{2} /\left(f_{0}^{2} Q^{2}\right)}},
$$

where the cantilever is assumed to be a damped harmonic oscillator excited at the frequency $f_{\text {exc }}$ with the amplitude $A_{\text {exc }}$ [2]. From a fit of this formula to a measured resonance curve, the fit parameters $f_{0}$ and $Q$ can be obtained.

There are several mechanisms contributing to the damping of an oscillating cantilever. The reciprocal of the effective $Q$ factor $1 / Q_{\text {eff }}$ describes the total damping, which is determined by the intrinsic damping $1 / Q_{0}$ of the cantilever, the damping $1 / Q_{\text {mount }}$ of the cantilever fixation in the force microscope and

6 Omicron NanoTechnology, Taunusstein, Germany.

7 Nanoworld, Neuchâtel, Switzerland.
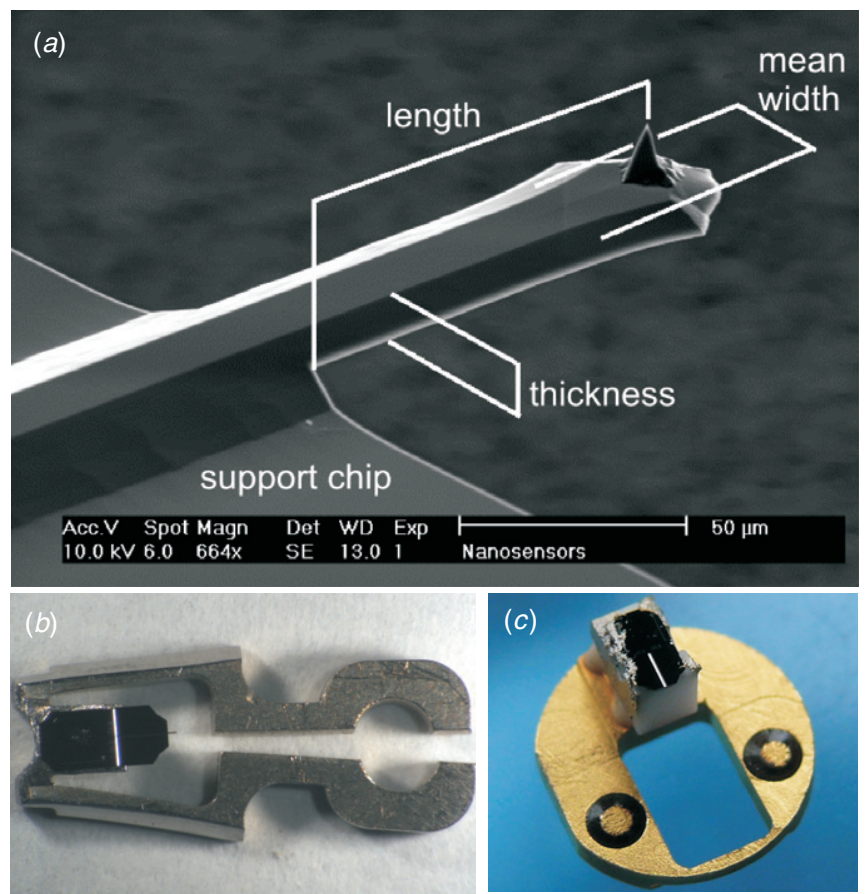

Figure 1. (a) Scanning electron microscopy micrograph of a cantilever on its support chip. Superimposed is a description of the cantilever geometric parameters: thickness $t$, width $w$ and length $l$.

(b) Photograph of the cantilever holder used in system A.

(c) Photograph of the cantilever holder used in system B. For both systems, the cantilever support chip is glued to the cantilever holder.

the air damping $1 / Q_{\text {air }}$, which needs to be considered when experiments are not performed under UHV conditions:

$$
\frac{1}{Q_{\text {eff }}}=\frac{1}{Q_{0}}+\frac{1}{Q_{\text {mount }}}+\frac{1}{Q_{\text {air }}} \text {. }
$$

The intrinsic $Q$-factor can be represented as the sum of the following major contributions [21]:

$$
\frac{1}{Q_{0}}=\frac{1}{Q_{\mathrm{vol}}}+\frac{1}{Q_{\text {support }}}+\frac{1}{Q_{\mathrm{TED}}}+\frac{1}{Q_{\text {surf }}},
$$

which are the volume loss $1 / Q_{\text {vol }}$, support loss $1 / Q_{\text {support }}$, thermoelastic damping $1 / Q_{\mathrm{TED}}$ and surface loss $1 / Q_{\text {surf }}$. The effective $Q$-factor cannot exceed the value of the smallest $Q$ contribution. A quantitative determination of all contributions to the damping is most difficult; however, for practical purposes it is sufficient to focus on the dominant damping mechanisms, namely support loss, surface damping and thermoelastic damping. The term $1 / Q_{\text {vol }}$ can be neglected as commercial standard cantilevers are made from monocrystalline material of highest purity.

Therefore, we consider the following contributions to the effective $Q$-factor:

$$
\frac{1}{Q_{\text {eff }}} \approx \frac{1}{Q_{\text {support }}}+\frac{1}{Q_{\mathrm{TED}}}+\frac{1}{Q_{\text {surf }}}+\frac{1}{Q_{\text {mount }}}+\frac{1}{Q_{\text {air }}} .
$$

Support loss determining $Q_{\text {support }}$ is the vibration energy of the cantilever dissipated by transmission through the support chip to which it is firmly attached (see figure 1(a)) [22]. The excited cantilever beam exerts both oscillating shear force and moment on its clamped end that, in turn, excite elastic 
Table 1. Contributions to the $Q$-factor calculated from the models described in the text for typical cantilever dimensions $(l=225 \mu \mathrm{m}$, $w=28 \mu \mathrm{m}, t=3 \mu \mathrm{m}$ for $75 \mathrm{kHz}$ cantilevers and $l=125 \mu \mathrm{m}, w=30 \mu \mathrm{m}, t=3.6 \mu \mathrm{m}$ for $300 \mathrm{kHz}$ cantilevers). $Q$-factors refer to a situation where cantilevers are kept at room temperature and $Q_{\text {surf }}$ is calculated assuming $\delta \times E_{2}^{S}=0.06$ (see section 3.3).

\begin{tabular}{rrrllr}
\hline$f_{0}(\mathrm{~Hz})$ & \multicolumn{1}{c}{$Q_{\text {support }}$} & \multicolumn{1}{c}{$Q_{\text {TED }}$} & $\left(Q_{\text {TED }}^{-1}+Q_{\text {support }}^{-1}\right)^{-1}$ & \multicolumn{1}{l}{$Q_{\text {surf }}$} & \multicolumn{1}{c}{$Q_{0}$} \\
\hline 75000 & 878000 & 1038000 & 476000 & 1334000 & 351000 \\
300000 & 87100 & 180000 & 58700 & 1594000 & 56700 \\
\hline
\end{tabular}

waves propagating into the support chip where their energy is dissipated. An analytical model for support loss in a micromachined beam resonator fixed at one end and free at the other with in-plane flexural vibrations presented by Hao et al [22] yields

$$
Q_{\text {support }}=2.081 \times\left(\frac{l}{t}\right)^{3},
$$

and we use this model to describe the support loss of cantilevers.

The thermoelastic damping (TED) in cantilevers was studied by Zener already in 1937 [23] and TED in silicon cantilevers was afterwards investigated by several groups [24-26]. Any bending of the cantilever is related to temperature changes, and an irreversible flow of heat driven by the generated temperature gradients gives rise to $Q_{\mathrm{TED}}$ [27]. For a cantilever made of perfect material with a nonzero thermal expansion coefficient $\alpha$, the energy dissipation caused by TED determines the upper limit for the intrinsic $Q$ factor. The contribution of TED can be derived approximately as [24]

$$
Q_{\mathrm{TED}}=\frac{\rho C_{p}}{E \alpha^{2} T} \frac{1+\left(2 \pi f_{0} \tau\right)^{2}}{2 \pi f_{0} \tau}
$$

with

$$
\tau=\frac{\rho C_{p} t^{2}}{\pi^{2} \kappa_{\mathrm{th}}}
$$

with the Young modulus $E$, density $\rho$, thermal conductivity $\kappa_{\text {th }}$ and specific heat capacity $C_{p}$ referring to a cantilever in equilibrium with a bath of temperature $T$. Lifshitz and Roukes derived a refined solution for TED which we will use in the following [27]:

$$
Q_{\mathrm{TED}}=\frac{\rho C_{p}}{E \alpha^{2} T}\left[\frac{6}{\xi^{2}}-\frac{6}{\xi^{3}} \times \frac{\sinh (\xi)+\sin (\xi)}{\cosh (\xi)+\cos (\xi)}\right]^{-1}
$$

with

$$
\xi=t \sqrt{\frac{2 \pi f_{0} \rho C_{p}}{2 \kappa_{\mathrm{th}}}} .
$$

The damping $1 / Q_{\text {surf }}$ caused by the surface layer of the cantilever can be expressed as [28]

$$
Q_{\text {surf }}=\frac{w t}{2 \delta(3 w+t)} \frac{E}{E_{2}^{S}}
$$

introducing the thickness $\delta$ of the surface layer and a parameter $E_{2}^{S}$ being a property of the surface layer and its defects [29]. Yang et al observed an increase in the $Q$-factor by an order of magnitude when removing the oxide layer from ultra-thin cantilevers (thickness $\approx 170 \mathrm{~nm}$ ) [29]. For typical cantilevers investigated here (thickness $\approx 4 \mu \mathrm{m}$ ), the effect of surface damping is, however, about 20 times smaller than for ultrathin cantilevers.

As will be demonstrated below, $Q_{\text {mount }}$ can be the most important contribution; however, it cannot be described by a simple formula as it depends on details of the mechanical contact of the cantilever support chip with the holder or of the cantilever holder with the body of the force microscope. The contact is influenced by subtleties that are difficult to describe mathematically and difficult to control practically. When the intrinsic damping characteristics $Q_{0}$ of the cantilever are known, $Q_{\text {mount }}$ can be obtained from the difference between theoretical $Q_{0}$ and experimental $Q_{\text {eff }}$ and taken as a measure for the quality of the cantilever fixation in the AFM:

$$
\frac{1}{Q_{\text {mount }}} \approx \frac{1}{Q_{\text {eff }}}-\frac{1}{Q_{0}} \text {. }
$$

In the case of an optimum fixation of the cantilever, $1 / Q_{\text {mount }}$ is zero.

The dependence of the $Q$-factor of silicon cantilevers on the pressure in the ambient gas has been investigated by Bianco et al [30] as well as by Blom et al [20] for certain ranges of the ambient pressure. It is possible to distinguish between pressure regimes that are dominated by different damping mechanisms, namely the molecular flow regime and the viscous flow regime [20]. For measurements performed under UHV conditions, only the molecular flow regime is relevant. For this regime, the pressure-dependent $Q$-factor is calculated based on models derived by Christian and Bianco $[30,31]$ :

$$
Q_{\text {molecular }}=\frac{\rho t \omega_{0}}{4} \sqrt{\frac{\pi}{2}} \sqrt{\frac{R T}{M}} \frac{1}{p},
$$

with the mass of the gas molecules $M$, the gas constant $R$, the temperature $T$ and the pressure $p$ of the gas. From this formula we find that $Q_{\text {molecular }}$ exceeds $10^{12}$ if the pressure is below $10^{-8}$ mbar. Therefore, this contribution is considered to be negligible in the context of this study.

To illustrate the importance of different contributions to the cantilever damping, we compile respective results in table 1. Using the known material properties of silicon cantilevers and typical dimensions, we calculate $Q_{0}$ considering the contributions of $Q_{\text {support }}, Q_{\mathrm{TED}}$ and $Q_{\text {surf }}$ for the two types of cantilevers used here. $Q_{\text {surf }}$ is estimated by a procedure described in detail in section 3.3.

Two major conclusions can be drawn from table 1. First, the $Q$-factor is principally limited to about 350000 for $75 \mathrm{kHz}$ cantilevers and to about 57000 for $300 \mathrm{kHz}$ cantilevers mainly due to the cantilever geometry. Second, there is limited room for an improvement of performance for the higher $Q$ cantilevers $(75 \mathrm{kHz})$ by reducing surface losses, while this contribution does not play any role for the lower $Q$ cantilevers $(300 \mathrm{kHz})$. 


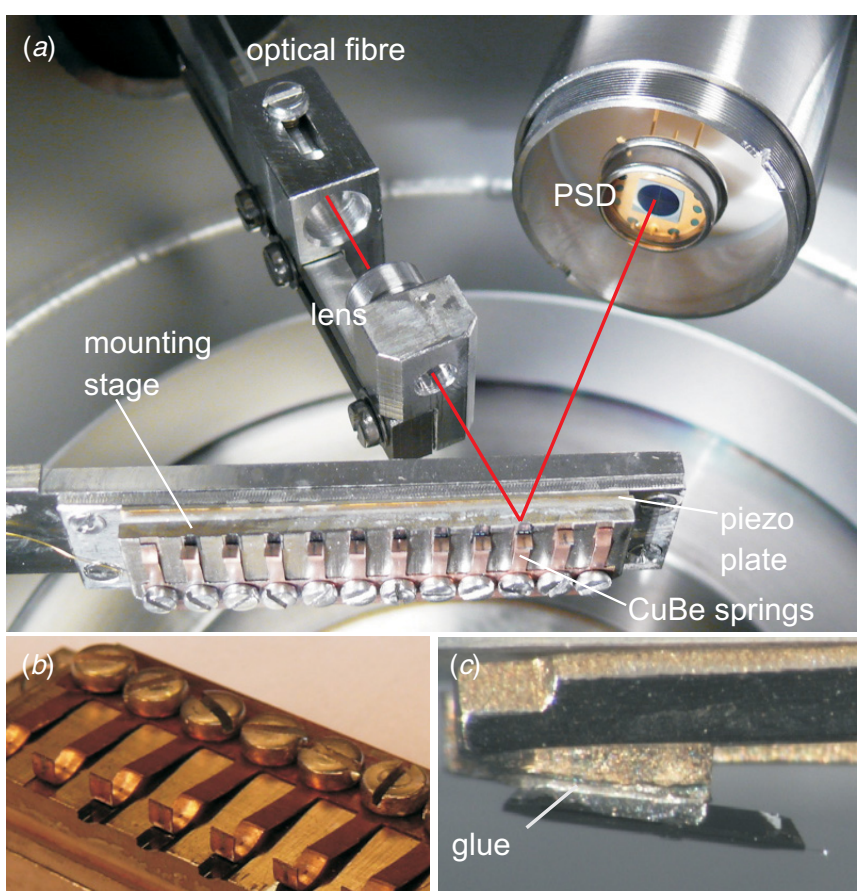

Figure 2. (a) View of the test stage for the measurement of $Q$-factors in UHV. Cantilevers are clamped to the mounting stage with $\mathrm{CuBe}$ springs and excited to mechanical oscillation by a piezo plate driven by a sinusoidal voltage at the desired frequency. The oscillation of the cantilever is detected by the deflection of a laser beam delivered by an optical fibre and focused onto the cantilever back side by an in-vacuo lens. The deflection is detected by a quadrant photosensitive detector. (b) Detail photo illustrating the spring clamping mechanism used in the test stage. (c) Illustration of gluing a cantilever support chip to the cantilever holder of system $\mathrm{A}$ for comparison.

\section{Experimental details}

The oscillation behaviour of cantilevers is investigated either in situ in one of the force microscopy systems A or B or in a test setup that is described below. The fixation mechanisms are different for the force microscopy systems. In system A, the holder consists of a V-shaped spring clamped into a counterpart on the AFM stage (see figure 1(b)). The cantilever holder of system B is positioned by three legs fitting into a socket and the holder is additionally fixed magnetically on the socket in the AFM stage (see figure 1(c)). In the test setup, cantilevers are mounted with a clamp fixation (see figures $2(a)$ and $(b))$ so that they can easily be removed and later be glued onto a cantilever holder, which is introduced into the AFM setup. All measurements reported here are performed with a system in thermal equilibrium at room temperature.

The test setup is housed in a compact vacuum chamber equipped with a turbomolecular pump and an ion getter pump. A combined Pirani/cold-cathode vacuum gauge allows us to measure the pressure from normal to UHV conditions. The detection of the cantilever oscillation is realized by the beam deflection method similar to the optical systems of our NC-AFM systems. Details of the test setup are shown in figure 2(a). A laser beam with a wavelength of $635 \mathrm{~nm}$ provided by a laser diode ${ }^{8}$ is coupled into the UHV by a single-mode optical fibre ${ }^{9}$ and focused by an in-vacuo lens (10 $\mathrm{mm}$ focal length) onto the backside of the cantilever. From there, the beam is reflected onto a four-quadrant photosensitive detector $(\mathrm{PSD})^{10}$. The photodiode has a spectral sensitivity of $0.5 \mathrm{~A} \mathrm{~W}^{-1}$ at the used wavelength yielding a typical photocurrent in the order of $0.1 \mathrm{~mA}$. Current signals from the quadrants are amplified and converted to voltage signals by home-built vacuum compatible pre-amplifiers directly attached to the four-quadrant diode. The deflection signal is generated by a difference amplifier processing the pairwise added voltage signals from the quadrants. The optical system of the test setup is similar to the setup used by system A, where the beam is deflected in a plane perpendicular to the plane defined by the incoming and reflected laser beams.

Cantilevers to be examined are mounted on a stage capable of holding up to 12 cantilevers. The cantilevers are aligned by milled recesses and fixed with copper-beryllium springs (see figure 2(a)), which allow for reuse of the cantilevers in a force microscope after the initial characterization. Details of the spring clamping mechanism are shown in figure $2(b)$. The cantilever mounting stage is equipped with a linear drive to select 1 out of the 12 cantilevers for the measurement. Precise alignment in the optical path is facilitated by an $\mathrm{X}-\mathrm{Y}$ positioning table. The base plate of the mounting stage is glued to a piezo ceramic plate with gold electrodes ${ }^{11}$ that is excited to vibration by applying an ac voltage of typically $0.1 \mathrm{mV}$ amplitude. For the replacement of cantilevers, the entire mounting stage is removed from the vacuum chamber.

To measure the $Q$-factor, we use a sine wave generator to excite the cantilever at a certain excitation amplitude $A_{\text {exc }}$ and sweep the frequency $f_{\text {exc }}$ in a range centred on the resonance frequency $f_{0}$ of the cantilever. A lock-in amplifier records the deflection signal as a function of the excitation frequency. A frequency sweep collecting 6400 data points is typically performed in $200 \mathrm{~s}$. The resonance frequency $f_{0}$ and the $Q$-factor $Q$ are obtained from the frequency spectrum by a least-squares fit of equation (3) to the data.

A typical resonance curve together with a fit result is displayed in figure 3. Measured values representing data obtained under UHV conditions can be well described by equation (3) for $f_{0}=67412.0 \mathrm{~Hz}$ and $Q=193700$. For the class of high- $Q 75 \mathrm{kHz}$ cantilevers, the width of the sweep is chosen to be $6 \mathrm{~Hz}$ or less to obtain a sufficiently high density of data points in the range of the maximum to facilitate a good fit. $Q$-factors can be determined with an accuracy of $2 \%$ taking into account errors due to changes of parameters such as ambient temperature, excitation amplitude, laser light intensity or adjustment of the optical system. However, we find that the measured $Q$-factor is most sensitive to the precise fixation of the cantilever support chip on the cantilever holder as will be discussed in the following section.

\footnotetext{
8 58FCM, Schäfter und Kirchhoff, Hamburg, Germany.

9 SMC-630, Schäfter und Kirchhoff, Hamburg, Germany.

10 JQ20P, Laser Components, Olching, Germany.

${ }^{11}$ EBL\#4, EBL Products Inc., East Hartford, CT, USA.
} 


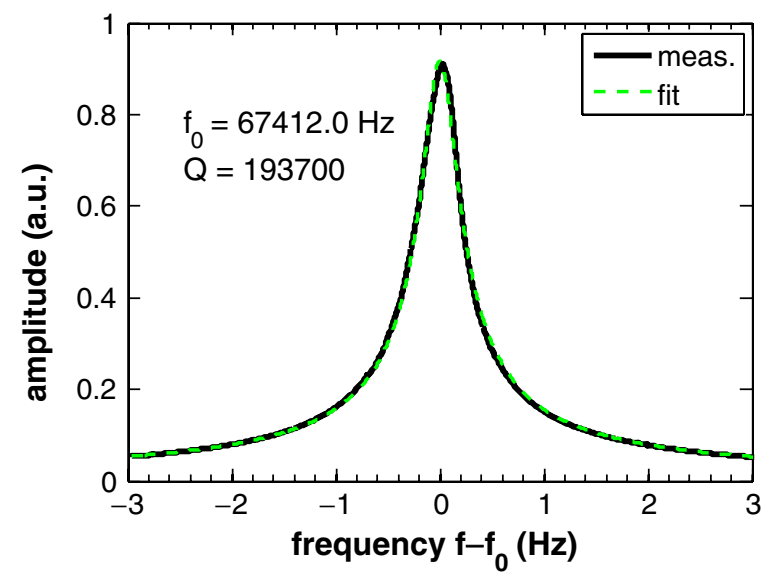

Figure 3. Measured resonance curve (solid line) with fit (dashed line) according to equation (3) yielding the fit parameters $f_{0}=67412.0 \mathrm{~Hz}$ and $Q=193700$.

\section{Results}

\subsection{Fluctuations of the effective $Q$-factor for clamped cantilevers}

We investigate the reproducibility of $Q$-factor measurements in the test setup. The $Q$-factors of a set of $75 \mathrm{kHz}$ cantilevers and of a set of $300 \mathrm{kHz}$ cantilevers are compared to each other. Each set consists of ten cantilevers taken from the same wafer. For $75 \mathrm{kHz}$ cantilevers as well as for $300 \mathrm{kHz}$ cantilevers, it appears that $Q$-factors cannot be measured with great reproducibility when cantilevers are removed from the cantilever holder and re-inserted, as evident from the diagrams shown in figure 4.

Frequently, we determine effective $Q$-factors that dramatically differ from $Q_{0}$, which is caused by additional damping due to peculiarities of mounting the cantilever. Measurement experience yields that a very large number of measurements is required to obtain $Q_{0}$ with a precision limited by the fit of the resonance curve, however, at least half of the measured effective $Q$-factors are fairly close to the real $Q$ factor of the cantilever. If an error margin of $20 \%$ of $Q_{0}$ is acceptable, it is enough to measure the $Q$-factor three times while removing and re-inserting the cantilever support chip into the cantilever holder between the measurements. The largest $Q$-factor measured for a cantilever is always assumed to be the best approximation to $Q_{0}$. Occasionally, this method fails as seen in figure 4 where cantilever no 20 exhibits a maximum $Q$-factor of 10900 when measured three times. A fourth measurement on this cantilever yields a $Q$-factor of 35100 , similar to all the other cantilevers from this batch. Having this cantilever inserted into system A, a value of 40100 is obtained (see cantilever 4 in figure 8 ).

Most of the cantilevers with a resonance frequency of $75 \mathrm{kHz}$ yield $Q_{0}$ values around 200000 , while the $300 \mathrm{kHz}$ cantilevers typically yield $Q_{0}$ values of 35000 . Results demonstrate that there are only minor variations of the $Q$ factor caused by the manufacturing process; however, large variations may result from the clamping fixation of the cantilevers in the mounting stage. This points to the general
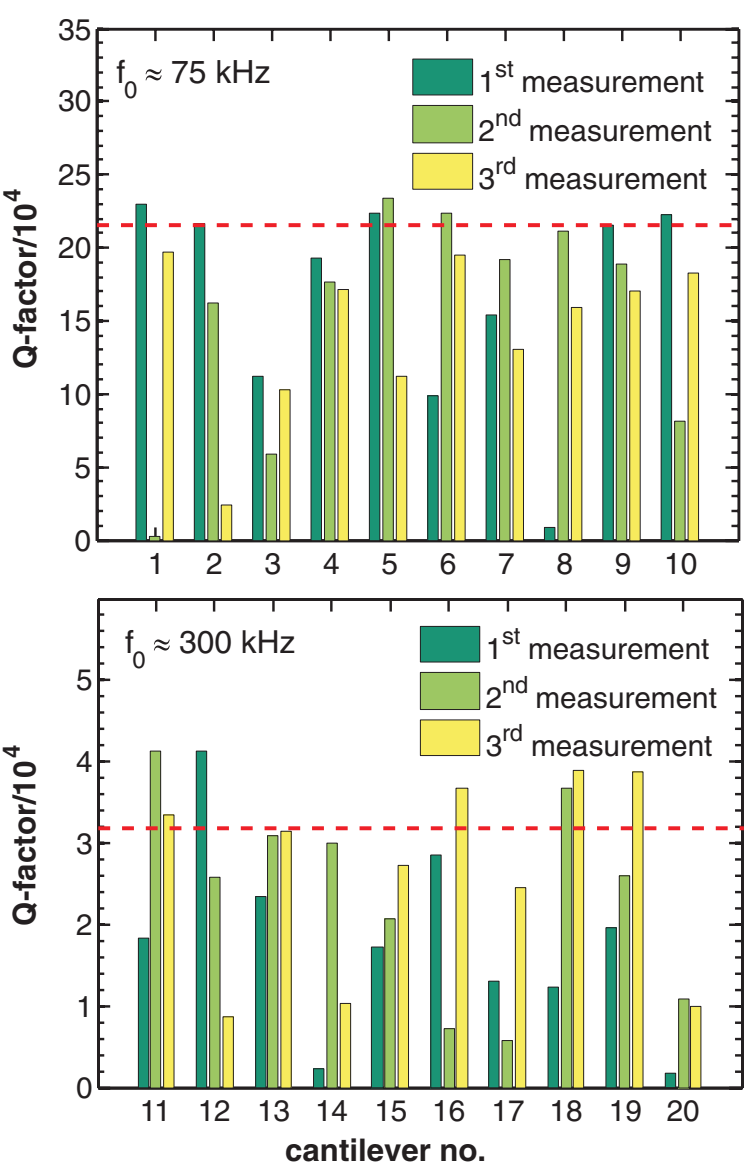

Figure 4. Test of reproducibility for the measurement of $Q$-factors of ten $75 \mathrm{kHz}$ cantilevers and ten $300 \mathrm{kHz}$ cantilevers in the test setup. Each cantilever is measured three times. Between the measurements, all cantilevers are removed from the mounting stage and re-inserted into a different slot (see figures $2(a)$ and $(b)$ ).

difficulty in reliably establishing a high- $Q$ oscillation with a cantilever that is clamped rather than glued to the cantilever holder. To test whether an improved result can be obtained by an optimized cantilever support, we equipped one slot of the stage with a cantilever alignment chip ${ }^{12}$ precisely fitting the cantilever support plate (see figure 5). Repetitive $Q$-factor measurements with this chip for two $75 \mathrm{kHz}$ cantilevers are shown in figure 6 . While generally the alignment chip provides a high reproducibility, also in this case some measurements yield dramatically reduced $Q$-factors. As one of the major causes of poor reproducibility, we identify small particles of silicon on the cantilever support chip prohibiting firm contact between mounting stage and cantilever support chip (see figure 7). These particles break off the chip when it is handled with tweezers to place the chip onto the mounting stage or when removing it. Possibly related observations have recently been reported as false resonance peaks of cantilevers operated in air where these peaks disappeared after readjusting the cantilever position [32]. In a more recent paper it has, furthermore, clearly been demonstrated that clamping a cantilever by a spring with a point or line contact likely results in a spurious response but a much cleaner frequency response

12 Alignment chip, version 4.2, Nanoworld, Neuchâtel, Switzerland. 


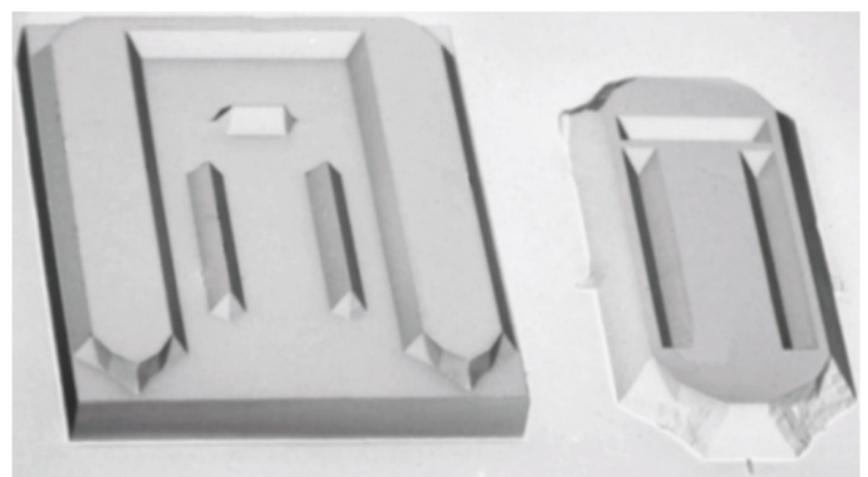

Figure 5. Cantilever alignment chip (left) precisely fitting to the backside of the cantilever support chip (right).

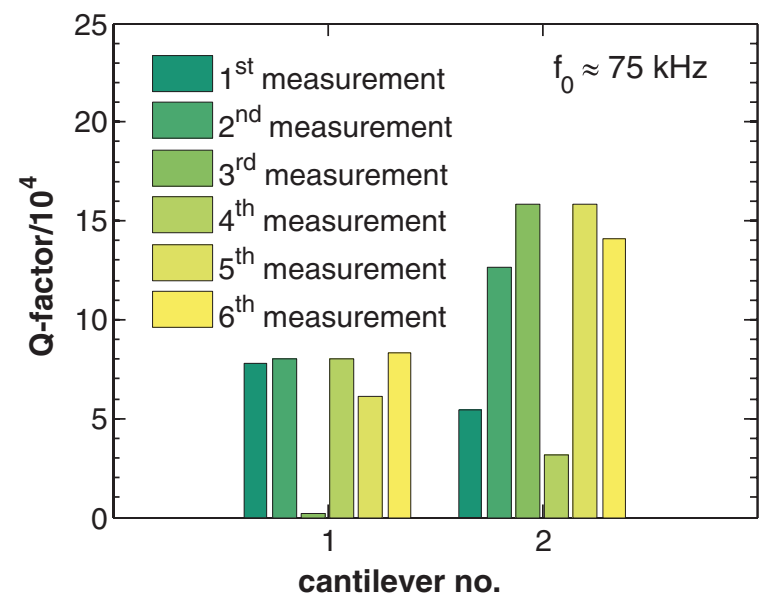

Figure 6. $Q$-factors of two cantilevers mounted onto the cantilever alignment chip measured in the test stage. Between measurements, the cantilever is removed and the alignment chip but not the cantilever support chip is cleaned with isopropyl alcohol before the cantilever is reinserted.

can be obtained when fixing the cantilever support chip with a rigid cover [33]. As will be evident from the following section, however, gluing the support chip on the cantilever holder is the most reliable method to obtain a high $Q$-factor.

\subsection{Fluctuations of the effective $Q$-factor for glued cantilevers}

We investigate how well $Q_{0}$ can be reproduced by effective $Q$-factors determined in the NC-AFM under realistic measurement conditions. In both force microscopes, the cantilever support chip is glued onto a removable cantilever holder (see figures $1(b)$ and $(c)$ ). For this type of fixation, a planar face of the cantilever holder is covered by a thin layer of glue and the cantilever support chip is pressed against the glue where it can be laterally positioned as long as the glue is not hardened. The final gluing result for a cantilever used in system A is shown in figure 2(c). We measure effective $Q$ factors in situ and test their reproducibility upon removal and re-insertion of the cantilever holder. Representative results for both systems are compiled in figure 8 .
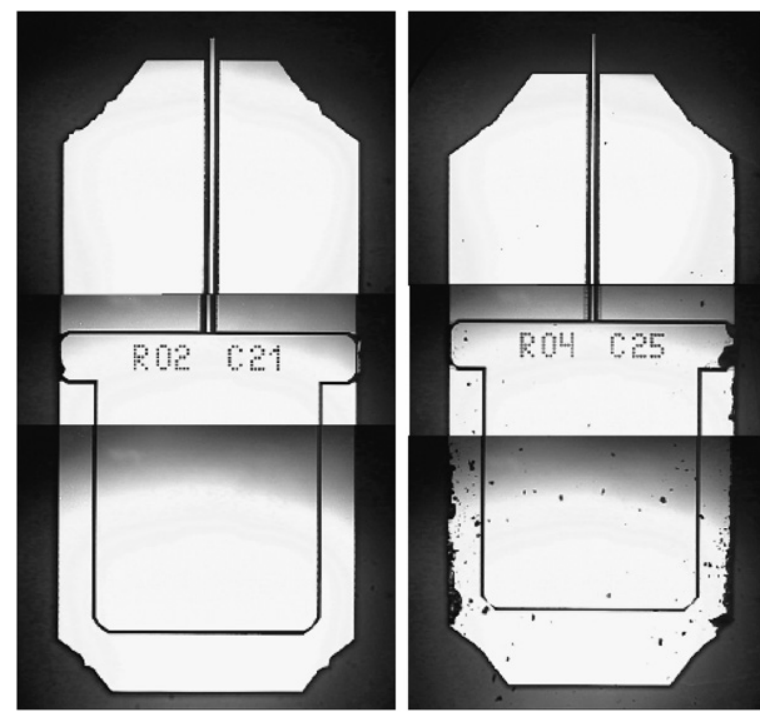

Figure 7. Left picture: image of the cantilever support chip of an unused AFM probe. Right picture: image of a similar cantilever support chip that has been handled with tweezers before mounting in the test setup. The dark dots represent small pieces of silicon broken off from the edges of the support chip.
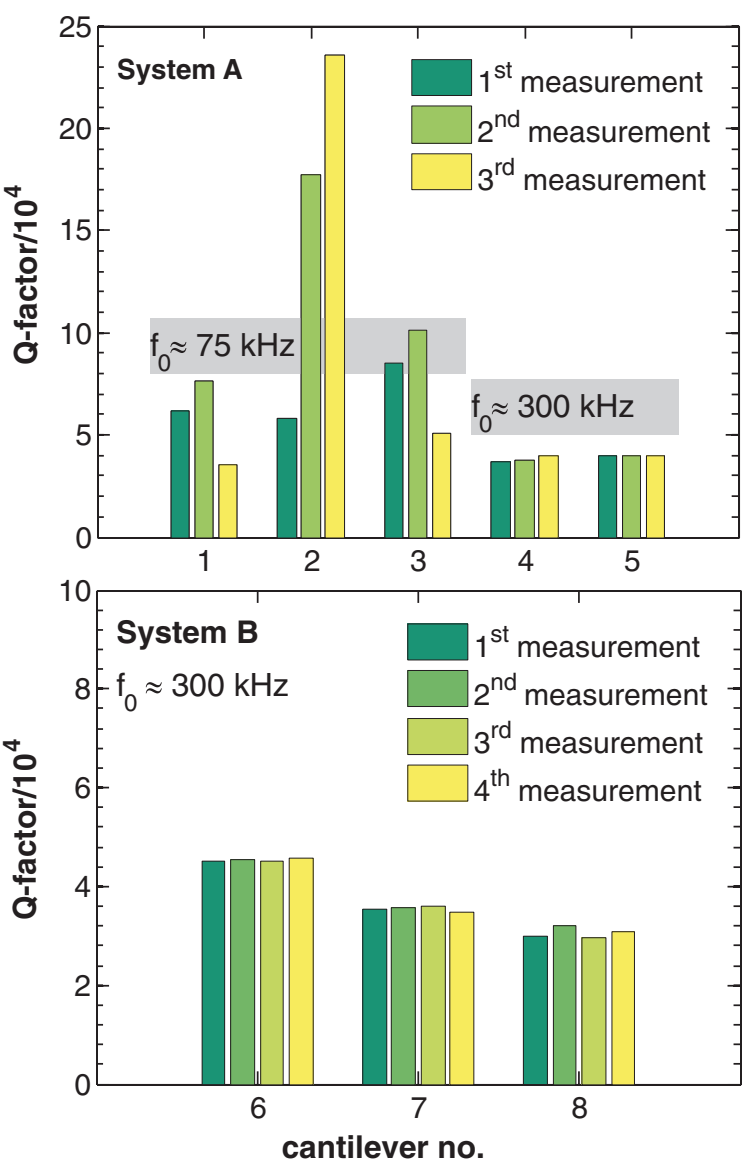

Figure 8. Measured $Q$-factors of cantilevers tested in NC-AFM systems A and B. Between individual measurements, the cantilever holder is removed and re-inserted into the AFM stage. 
Table 2. Estimated values for $Q_{\text {surf }}$ and $Q_{\text {mount }}$ based on the discrepancy between the calculated $\left(Q_{\mathrm{TED}}^{-1}+Q_{\text {support }}^{-1}\right)^{-1}$ and the measured $Q_{\text {eff }}^{\text {exp }}$. $Q_{0}^{\text {theo }}$ values are calculated according to equation (6) assuming $\delta \times E_{2}^{S}=0.06$ for all cantilevers and assuming that the additional damping is due to $Q_{\text {mount }}$.

\begin{tabular}{lllllllll}
\hline$t(\mu \mathrm{m})$ & $l(\mu \mathrm{m})$ & $w(\mu \mathrm{m})$ & $f_{0}^{\exp }(\mathrm{Hz})$ & $Q_{\mathrm{eff}}^{\exp }$ & $\left(Q_{\mathrm{TED}}^{-1}+Q_{\text {support }}^{-1}\right)^{-1}$ & $Q_{\text {surf }}$ & $Q_{0}^{\text {theo }}$ & $Q_{\text {mount }}$ \\
\hline 3.6 & 126 & 29 & 287555 & 43900 & 60000 & 1592000 & 58300 & 178000 \\
3.6 & 126 & 29 & 290529 & 44500 & 60300 & 1592000 & 58100 & 190000 \\
3.6 & 126 & 29 & 291104 & 40000 & 60300 & 1592000 & 58100 & 128000 \\
3.6 & 126 & 29 & 293719 & 40500 & 60100 & 1592000 & 57900 & 135000 \\
3.6 & 126 & 29 & 295600 & 43000 & 60000 & 1592000 & 57800 & 168000 \\
3.7 & 126 & 29 & 294810 & 31000 & 55800 & 1634000 & 54000 & 72900 \\
3.7 & 126 & 29 & 297730 & 40000 & 55600 & 1634000 & 53800 & 156000 \\
3.8 & 126 & 29 & 295707 & 39000 & 51900 & 1677000 & 50300 & 173000 \\
3.9 & 126 & 29 & 290280 & 44000 & 48700 & 1719000 & 47300 & 625000 \\
3.9 & 125 & 27 & 312450 & 36700 & 46800 & 1714000 & 45600 & 189000 \\
3.9 & 125 & 27 & 314597 & 45400 & 46700 & 1714000 & 45500 & $\infty$ \\
4.0 & 126 & 29 & 302990 & 38000 & 44900 & 1761000 & 43800 & 289000 \\
4.0 & 125 & 27 & 305193 & 41000 & 44000 & 1755000 & 43000 & 899000 \\
4.0 & 125 & 27 & 313739 & 36000 & 43700 & 1755000 & 42600 & 232000 \\
4.0 & 125 & 27 & 321802 & 32000 & 43300 & 1755000 & 42300 & 132000 \\
4.2 & 125 & 28 & 299087 & 30000 & 38800 & 1842000 & 38000 & 142000 \\
\hline
\end{tabular}

In system $\mathrm{A}$, a large variation of the $Q$-factor is found for the $75 \mathrm{kHz}$ cantilevers, while effective $Q$-factors for the $300 \mathrm{kHz}$ cantilevers remain nearly constant indicating that the $Q$-factors of the $75 \mathrm{kHz}$ cantilevers are more sensitive to the mounting mechanical contact. This can be understood as the intrinsic $Q$-factor of $300 \mathrm{kHz}$ cantilevers is significantly smaller than the intrinsic $Q$-factor of $75 \mathrm{kHz}$ cantilevers and, therefore, the relative effect of additional losses by mounting is smaller for $300 \mathrm{kHz}$ cantilevers. The high reproducibility of the effective $Q$-factor for $300 \mathrm{kHz}$ cantilevers is confirmed by measurements in system $\mathrm{B}$, also yielding only very small variations of the $Q$-factor. From a comparison of these results to those described in the previous section, we find that $Q$-factor fluctuations of cantilevers glued in the NC-AFM systems are much less than fluctuations observed for the test setup where cantilevers are clamped.

\subsection{Estimating surface damping}

Surface damping is the contribution to $Q_{0}$ that is most difficult to handle as the respective material parameters are neither tabulated nor can they be measured directly. As the parameters $\delta$ and $E_{2}^{S}$ controlling surface damping are not known, we fit equation (12) to the measured data using the product $\delta \times E_{2}^{S}$ as a fit parameter. We adopt the procedure of Hao et al who fit $\delta \times E_{2}^{S}$ to explain the discrepancy between $Q_{\mathrm{TED}}^{-1}+Q_{\text {support }}^{-1}$ and $Q_{\text {eff }}^{-1}$ by surface damping [22] ${ }^{13}$, however, extend this by including $Q_{\text {mount }}$ that we identified as a possibly most important contribution. We assume all uncoated silicon cantilevers investigated to be covered with a native oxide layer of the same thickness $\delta$ and the same property $E_{2}^{S}$. According to equation (6) we then obtain the equation

$$
\frac{1}{Q_{\text {mount }}}+\frac{1}{Q_{\text {surf }}}=\frac{1}{Q_{\text {eff }}}-\left(\frac{1}{Q_{\mathrm{TED}}}-\frac{1}{Q_{\text {support }}}\right),
$$

${ }^{13}$ Hao et al fitted equation (12) with the product $\delta \cdot E_{2}^{S}$ as a parameter to their measured data but have mistakenly interchanged cantilever height and width. where $Q_{\text {mount }}$ is an unknown contribution depending on the experimental details, while $Q_{\text {surf }}$ is a predictable contribution depending on the known cantilever geometry and the yet unknown parameter $\delta \times E_{2}^{S}$. To determine this parameter, we investigate 15 cantilevers from one batch that nominally have the same properties. For each of these cantilevers, we determine the resonance frequency $f_{0}^{\exp }$ and $Q_{\text {eff }}^{\exp }$ from the measurements in system B and determine $Q_{\mathrm{TED}}^{-1}+Q_{\text {support }}^{-1}$ from the measured dimensions and known material parameters. The respective results are compiled in columns 4, 5 and 6 of table 2 . According to equation (15) the difference between these two quantities provides the sum of the damping contributions of $Q_{\text {mount }}$ and $Q_{\text {surf }}$. From table 2 we find that the difference is negligible or small for some of the measurements and anticipate that this indicates a small or vanishing mounting loss. For the cantilever with the smallest difference, we assume $Q_{\text {mount }}^{-1}$ to vanish and associate the difference with $Q_{\text {surf }}^{-1}$. From the results of this measurement we can deduce the parameter $\delta \times E_{2}^{S}=0.06$ according to equation (12) and take this as a universal parameter not only for this batch of cantilevers but for all cantilevers $(300 \mathrm{kHz}$ and $75 \mathrm{kHz})$ produced under similar conditions. Using the parameter $\delta \times E_{2}^{S}$, we can calculate $Q_{\text {surf }}, Q_{\text {mount }}$ and the intrinsic damping $Q_{0}^{\text {theo }}$ where the respective results are shown as columns 7, 8 and 9 of table 2 . We find that $Q_{\text {surf }}$ is a small contribution for the investigated $300 \mathrm{kHz}$ cantilevers while $Q_{\text {mount }}$ is of the order of $Q_{\mathrm{TED}}$ or $Q_{\text {support }}$ except for a few cases where it greatly exceeds these contributions due to the perfect mounting of the cantilever. Any variation of measured $Q_{\text {eff }}^{\exp }$ for cantilevers with identical dimensions is explained by individual values of $Q_{\text {mount }}$.

\subsection{Maintaining a high effective $Q$-factor in the NC-AFM}

The figure-of-merit related to the cantilever oscillation properties relevant for high resolution NC-AFM measurements is $Q_{\text {eff }}$ as determined in the NC-AFM. To investigate to what extent high values for $Q_{\text {eff }}$ determined in the test setup can be maintained when using a cantilever in a NC-AFM system, 

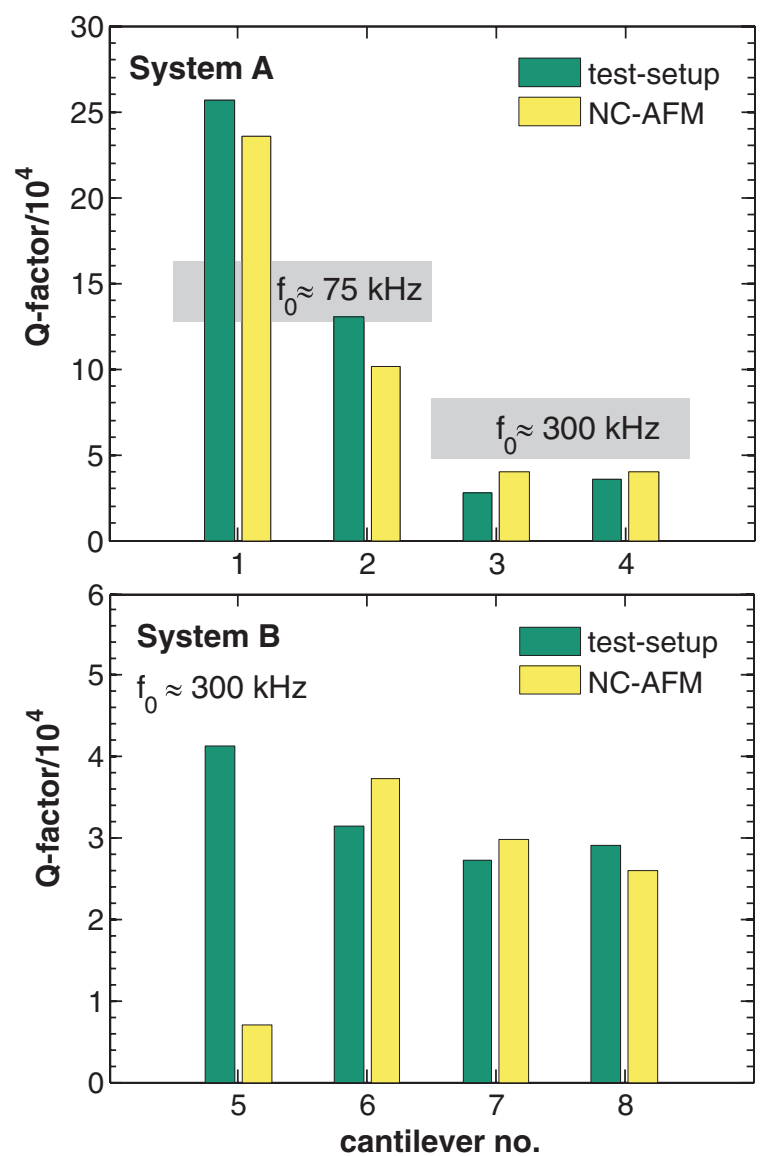

Figure 9. $Q$-factors of cantilevers measured in the test setup compared to the $Q$-factors of the same cantilevers measured in NC-AFM systems A and B.

we perform comparative measurements on the same cantilever investigated in both environments.

After having been studied in the test setup, cantilevers are glued onto cantilever holders of the AFM systems (see figure 2(c) as an example). In all cases, the same conductive silver-epoxy glue is used ${ }^{14}$; however, the thickness of the glue film under the cantilever support chip may differ. Results are compiled in figure 9. In both systems, the highest value of multiple measurements was taken to minimize mounting effects.

In system $\mathrm{A}$, results show a slight decrease in the $Q$-factors of the $75 \mathrm{kHz}$ cantilevers and a slight increase in the $Q$-factors of the $300 \mathrm{kHz}$ cantilevers when moved from the test setup to the NC-AFM. For this cantilever holder, we find that the glue has only a small influence on the $Q$-factor while the fixation of the cantilever holder in the scan head can result in some change of the $Q$-factor (see cantilever 2 in figure 8 ).

In system $\mathrm{B}$, we generally find highest reproducibility; however, peculiarities in gluing the cantilever may exceptionally result in a dramatic reduction of the effective $Q$-factor as observed for cantilever 5 in figure 9 .

These results demonstrate that it is possible to routinely exploit the high intrinsic $Q$-factors of commercially available cantilevers provided care is taken in gluing and mounting

\footnotetext{
${ }^{14}$ Epotek H21D, Polytec PT, Waldbronn, Germany.
}

Table 3. Minimum detectable force gradient $\delta F_{\min }^{\prime}$ for typical cantilever properties $f_{0}, k$ and $Q$ as resulting from equation (1). The $Q$-factors are typical effective and best values measured for the respective types of cantilevers. Other parameters: temperature $T=300 \mathrm{~K}$, detection bandwidth $B=300 \mathrm{~Hz}$, oscillation amplitude $A=10 \mathrm{~nm}$.

\begin{tabular}{rrrrl}
\hline$f_{0}$ & \multicolumn{1}{c}{$k$} & \multicolumn{1}{c}{$Q_{\text {eff }}^{\text {typical }}$} & \multicolumn{1}{l}{$Q_{\text {eff }}^{\text {best }}$} & \multicolumn{1}{l}{$\delta F_{\text {min }}^{\prime}$} \\
\hline $75 \mathrm{kHz}$ & $2.8 \mathrm{~N} \mathrm{~m}^{-1}$ & 200000 & 300000 & $1.4 \times 10^{-6} \mathrm{~N} \mathrm{~m}^{-1}$ \\
$300 \mathrm{kHz}$ & $42.0 \mathrm{~N} \mathrm{~m}^{-1}$ & 35000 & 45000 & $7.0 \times 10^{-6} \mathrm{~N} \mathrm{~m}^{-1}$ \\
\hline
\end{tabular}

them. Best values obtained for $Q_{\text {eff }}$ are 300000 for $75 \mathrm{kHz}$ cantilevers and 45000 for $300 \mathrm{kHz}$ cantilevers. From a total of 50 measurements with $300 \mathrm{kHz}$ cantilevers from one batch, we find mean $Q_{\text {mount }}$ values of 68000 for the test setup, 198000 for system A and 263000 for system B. This implies that $Q_{\text {eff }}$ has to be measured in the respective system prior to any critical experiment where highest performance is required. Apparently, system B exhibits the smallest mounting loss on average and is, therefore, used as the reference system for in situ studies for the present work.

The values given above determine the principal limits of the force detection sensitivity for cantilever-based NC-AFM measurements performed under UHV conditions. In table 3, the minimum detectable force gradients calculated from equation (1) are given for $75 \mathrm{kHz}$ and $300 \mathrm{kHz}$ cantilevers assuming routinely observed $Q$-factors and operation at room temperature. We find a minimum detectable force gradient better than $10^{-5} \mathrm{~N} \mathrm{~m}^{-1}$ where $75 \mathrm{kHz}$ cantilevers yield half an order of magnitude more force sensitivity than $300 \mathrm{kHz}$ cantilevers. Practically, the sensitivity will in most systems be reduced by the noise from the light source, pre-amplifier and demodulation electronics used in the NC-AFM system.

\section{Conclusions}

Commercially available high quality cantilevers generally have high intrinsic $Q$-factors; typical values are 200000 for $75 \mathrm{kHz}$ cantilevers and 35000 for $300 \mathrm{kHz}$ cantilevers, while the best obtained values are 300000 and 45000 , respectively. The best measured $Q$-factors relevant for NCAFM measurements in UHV are close to the intrinsic values; however, the $Q$-factor may be strongly reduced by mounting the cantilever. Gluing the cantilever to the holder is the most reliable method to yield a high effective $Q$-factor, while clamping yields large fluctuations unless utmost care in positioning the cantilever and cleanliness of cantilever and holder is taken. Taking the necessary care in mounting the cantilever, it is possible to routinely obtain an effective $Q$ factor in NC-AFM measurements in high or ultra-high vacuum that is within a $20 \%$ margin of the intrinsic $Q$-factor. However, for critical measurements, this has to be verified by a $Q$-factor measurement with the cantilever mounted in the NC-AFM system and cannot be inferred from any ex situ measurement. The $Q$-factor that realistically can be expected in a NC-AFM system defines a principal limit of the minimum detectable force gradient for room temperature measurements in the order of $10^{-6} \mathrm{~N} \mathrm{~m}^{-1}$. 


\section{Acknowledgments}

We are grateful to Hans-Hermann Pieper, Philipp Rahe and Jens Schütte for supporting the measurements in the different NC-AFM systems.

\section{References}

[1] Morita S, Wiesendanger R and Meyer E 2002 Noncontact Atomic Force Microscopy (Nanoscience and Technology) (Berlin: Springer)

[2] Giessibl F J 2003 Advances in atomic force microscopy Rev. Mod. Phys. 75 949-83

[3] Reichling M and Barth C 1999 Scanning force imaging of atomic size defects on the $\mathrm{CaF}_{2}$ (111) surface Phys. Rev. Lett. 83 768-71

[4] Barth C and Reichling M 2001 Imaging the atomic arrangements on the high-temperature reconstructed alpha- $\mathrm{Al}_{2} \mathrm{O}_{3}$ (0001) surface Nature 414 54-7

[5] Barth C, Foster A S, Reichling M and Shluger A L 2001 Contrast formation in atomic resolution scanning force microscopy on $\mathrm{CaF}_{2}$ (111): experiment and theory J. Phys.: Condens. Matter 13 2061-79

[6] Gritschneder S, Namai Y, Iwasawa Y and Reichling M 2005 Structural features of $\mathrm{CeO}_{2}$ (111) revealed by dynamic SFM Nanotechnology 16 S41-8

[7] Hirth S, Ostendorf F and Reichling M 2006 Lateral manipulation of atomic size defects on the $\mathrm{CaF}_{2}$ (111) surface Nanotechnology 17 S148-54

[8] Torbrügge S, Reichling M, Ishiyama A, Morita S and Custance O 2007 Evidence of subsurface oxygen vacancy ordering on reduced $\mathrm{CeO}_{2}$ (111) Phys. Rev. Lett. 99056101

[9] Torbrügge S, Ostendorf F and Reichling M 2009 Stabilization of zinc-terminated $\mathrm{ZnO}(0001)$ by a modified surface stoichiometry J. Phys. Chem. C 113 4909-14

[10] Ostendorf F, Schmitz C, Hirth S, Kühnle A, Kolodziej J J and Reichling M 2009 Evidence for potassium carbonate crystallites on air-cleaved mica surfaces Langmuir 25 10764-7

[11] Albrecht T R, Grütter P, Horne D and Rugar D 1991 Frequency modulation detection using high- $Q$ cantilevers for enhanced force microscopy sensitivity J. Appl. Phys. 69 668-73

[12] Kikukawa A, Hosaka S and Imura R 1996 Vacuum compatible high-sensitive Kelvin probe force microscopy Rev. Sci. Instrum. 67 1463-7

[13] Kawai S, Kitamura S, Kobayashi D, Meguro S and Kawakatsu H 2005 An ultrasmall amplitude operation of dynamic force microscopy with second flexural mode Appl. Phys. Lett. 86193107

[14] Sadewasser S, Villanueva G and Plaza J A 2006 Special cantilever geometry for the access of higher oscillation modes in atomic force microscopy Appl. Phys. Lett. 89033106

[15] Girard P, Ramonda M and Arinero R 2006 Dynamic atomic force microscopy operation based on high flexure modes of the cantilever Rev. Sci. Instrum. 77096105
[16] Paulo A S, Black J P, White R M and Bokor J 2007 Detection of nanomechanical vibrations by dynamic force microscopy in higher cantilever eigenmodes Appl. Phys. Lett. 91053116

[17] Baumann M and Stark R W 2010 Dual frequency atomic force microscopy on charged surfaces Ultramicroscopy 110 578-81

[18] Howald L, Meyer E, Lüthi R, Haefke H, Overney R, Rudin H and Güntherodt H J 1993 Multifunctional probe microscope for facile operation in ultrahigh-vacuum Appl. Phys. Lett. 63 117-9

[19] Torbrügge S, Lübbe J, Tröger L, Cranney M, Eguchi T, Hasegawa Y and Reichling M 2008 Improvement of a dynamic scanning force microscope for highest resolution imaging in ultrahigh vacuum Rev. Sci. Instrum. 79083701

[20] Blom F R, Bouwstra S, Elwenspoek M and Fluitman J H J 1992 Dependence of the quality factor of micromachined silicon beam resonators on pressure and geometry J. Vac. Sci. Technol. B 10 19-26

[21] Naeli K and Brand O 2009 Dimensional considerations in achieving large quality factors for resonant silicon cantilevers in air J. Appl. Phys. 105014908

[22] Hao Z, Erbil A and Ayazi F 2003 An analytical model for support loss in micromachined beam resonators with in-plane flexural vibrations Sensors Actuators A 109 156-64

[23] Zener C 1937 Internal friction in solids: I. Theory of internal friction in reeds Phys. Rev. 52 230-5

[24] Srikar V T and Senturia S D 2002 Thermoelastic damping in fine-grained polysilicon flexural beam resonantors J. Microelectromech. Syst. 11 499-504

[25] Gysin U, Rast S, Ruff P, Meyer E, Lee D W, Vettiger P and Gerber C 2004 Temperature dependence of the force sensitivity of silicon cantilevers Phys. Rev. B 69045403

[26] Candler R N et al 2006 Impact of geometry on thermoelastic dissipation in micromechanical resonant beams J. Microelectromech. Syst. 15 927-34

[27] Lifshitz R and Roukes M L 2000 Thermoelastic damping in micro- and nanomechanical systems Phys. Rev. B 61 5600-9

[28] Yasumura K Y, Stowe T D, Chow E M, Pfafman T, Kenny T W, Stipe B C and Rugar D 2000 Quality factors in micron- and submicron-thick cantilevers J. Microelectromech. Syst. 9 117-25

[29] Yang J, Ono T and Esashi M 2002 Energy dissipation in submicrometer thick single-crystal silicon cantilevers J. Microelectromech. Syst. 11 775-83

[30] Bianco S et al 2006 Silicon resonant microcantilevers for absolute pressure measurement J. Vac. Sci. Technol. B 24 1803-9

[31] Christian R G 1966 The theory of oscillating-vane vacuum gauges Vacuum 16 175-8

[32] Adams J D, York D and Whisman N 2004 Reduction of spurious resonance peaks in microcantilever tuning through substrate contact surface reduction Rev. Sci. Instrum. 75 2903-5

[33] Tsuji T, Kobari K, Ide S and Yamanaka K 2007 Suppression of spurious vibration of cantilever in atomic force microscopy by enhancement of bending rigidity of cantilever chip substrate Rev. Sci. Instrum. 78103703 